\title{
Using integrated geophysical research Lala M163-1 aeromagnetic anomaly and ore prospect analysis, in Huili county, Sichuan province, China
}

\author{
Lin Qiu*, Xuben Wang \\ Key Lab of Earth Exploration and Information Techniques of Ministry of Education \\ Chengdu University of Technology \\ Chengdu 610059, China
}

\begin{abstract}
The previous person thought that the M163-1 aeromagnetic anomaly is caused by the concealed and magnetite content alteration gabbro, but through the study of regional gravity, magnetic data and geological data,we thought M163-1 aeromagnetic anomaly has the larger mineralization potential., which compare with Lala copper deposit M162 aeromagnetic anomaly. Therefore,the ground high-precision magnetic method and audio magnetotelluric sounding method are adopted to investigate the fine characteristics of magnetic anomaly and the deep electrical structure of Lala M163-1 aeromagnetic anomaly, which is combined with the regional magnetic data and geological data on abnormal area to carry out comprehensive geophysical verification work.Fine characteristic of magnetic anomaly and the deep electrical structure of Lala M163-1 are preliminarily determined by comprehensive analysis of regional gravity and magnetic,ground high precision magnetic and audio magnetotelluric sounding method. At last, We got two magnetic anomalies and relative high apparent resistivity anomalies, which are meaningful ore to anomaly. So, we got new knowing different from the previous person's about M163-1 aeromagnetic anomaly.This provides the important geophysical basis for the anomaly's ore-prospecting prospective study evaluation.
\end{abstract}

Key words - ground high-precision magnetic method, audio magnetotelluric sounding method, aeromagnetic anomaly, ore-prospecting prospective

\section{INTRODUCTION}

M163-1 aeromagnetic anomaly is located in about $9.0 \mathrm{~km}$ south of Lala copper mine in Huili County of sichuan province, and is located in the middle of the Kangdian Axis in geotectonics, which is important copper polymetallic metallogenic belt in China ${ }^{[1-6]}$. Regional gravity and magnetic data shows that Lala copper mine and its periphery is located in high magnetic and high gravity anomaly. There distribute larger scale ore deposit in M162 aeromagnetic anomaly, such as Luodang copper deposit, Tianshengba iron deposit and Shilong copper-iron deposit, etc; Then, in M163-1 aeromagnetic anomaly which is the same scale and intensity to M162, similar deposits are not found, there are only two smaller Guandi and Lijiafen iron mines in the north of M163-1. So, whether there are similar scale deposit in aeromagnetic anomaly M163-1 ? For this, the previous person arrange a drilling in the center of M163-1 aeromagnetic anomaly, and the verification shows that the anomaly reflects the concealed and magnetite content alteration gabbro ${ }^{[7]}$, since then, there was no detailed verification work. However, M163-1 is the same scale to M162, whether or not the conclusion conforms to the reality about M163-1 aeromagnetic anomaly, it remains to be further discussion and validation. Therefore, in order to understand the characteristics of fine magnetic anomaly and the deep electrical structure of M163-1 aeromagnetic anomaly, this paper adopts exploration pattern that combines the ground of high precision magnetic method with audio magnetotelluric sounding (AMT) to carry out comprehensive research, which is of great significance for the periphery prospecting perspective analysis of Lala copper mine, and provide geophysical basis for the next step prospecting work.

\section{GEOPHYSICAL SURVEY AND DATA PROCESSING}

According to the situation of measured area geological, the difference of physical property and exploration purpose, the ground high-precision magnetic method and audio magnetotelluric sounding method are adopted to carry out inspection work of M163 1 aeromagnetic anomaly. The ground high-precision magnetic method and AMT line on the same profile, are all through the center of M163-1 aeromagnetic anomaly. Section starts at the Guandi iron mines in the north, through the Lijiafen iron mines to the south, then through the M163-1 aeromagnetic anomaly, and end at Jiangyi stone mountain to the south.The total length of the section is $12 \mathrm{~km}$. The measurement point distance of magnetic and AMT are separately $20 \mathrm{~m}$ and $100 \mathrm{~m}$. Line azimuth is 
$20.31^{\circ}$ in the interval of -4000 to 3500 , and line azimuth is $10.75^{\circ}$ in the interval of 3500 to 8000 . As shown in Fig. 1.and the inversion interpretation. Firstly, use curve to identify and adjust the polarization mode, and then do curve smoothing processing, finally, do static correction processing on data. AMT inversion uses 1-D Bostic transform as the initial inversion model, and uses 2-D NLCG inversion as the final result; among which, Combine with the result of magnetic method in the process of inversion, to do appropriate correction for the initial inversion model,then to do the inversion processing again.

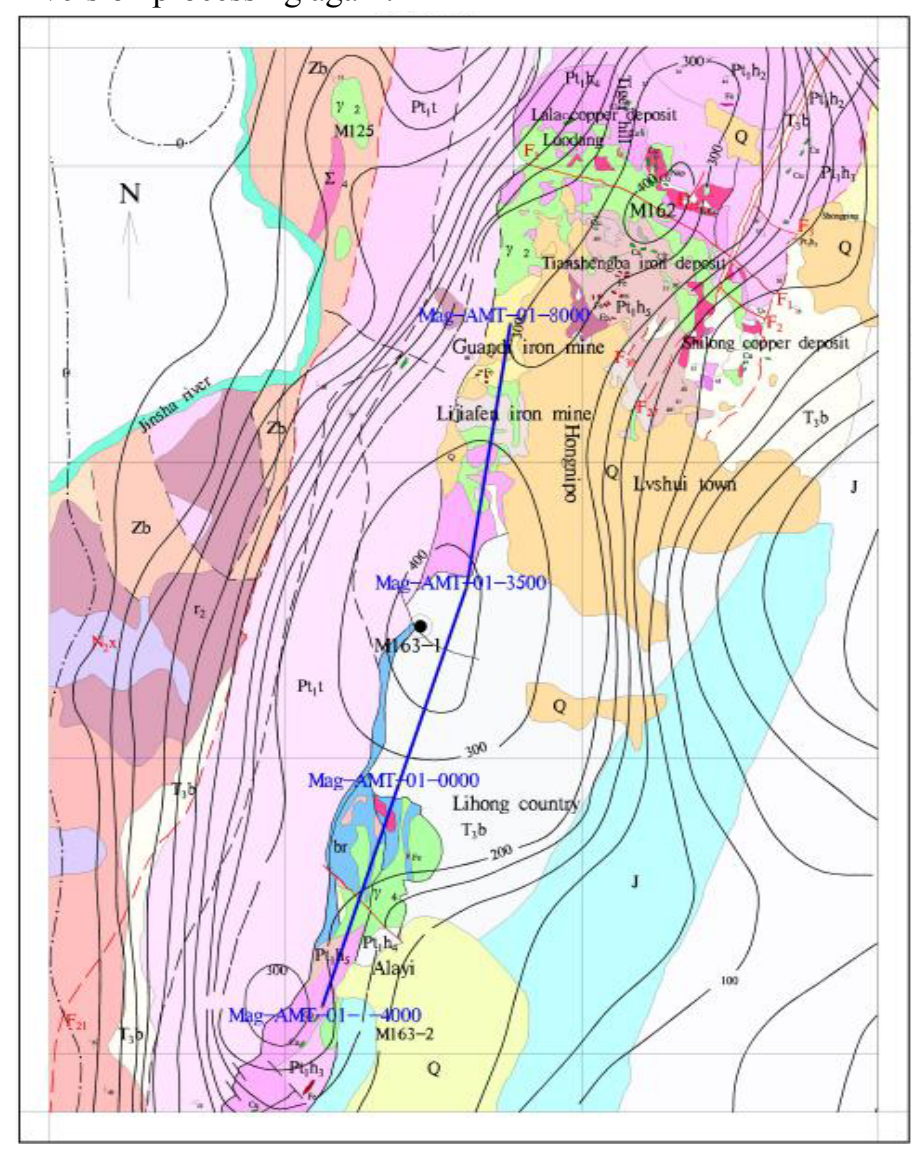

Fig. 1. Magnetic method and AMT line layout

\section{III.COMPREHENSIVE INTERPRETATION}

By processing the ground high-precision magnetic survey data and audio magnetotelluric sounding data, we have obtained two Secondary magnetic anomalies and relatively high resistivity anomalies in M163-1 aeromagnetic anomaly area, infered a fault F1 and a low resistance fracture zone F2, and divided the deep electrical structure preliminary. The structure characteristics of the section on the whole is syncline, which is coincide with the predecessors' thought. The interpretation result of the ground high-precision magnetic method and AMT profile data comprehensive anomaly is shown in Fig. 2.

According to the survey area aeromagnetic anomaly data, M163-1 aeromagnetic anomaly mainly distributed in the cross-sectional segment $0 \sim 6000 \mathrm{~m}$. The ground precision magnetic measurement results of FIG. 2 in the region also showed higher magnetic anomaly. But compared with the aeromagnetic, ground magnetic anomaly survey is different, it will be more finely to divide M163-1 aeromagnetic anomaly into two smaller secondary magnetic anomaly, which was showed in FIG. 2, $\Delta \mathrm{T}-1$ and $\Delta \mathrm{T}-2$, after reduced to the pole; $\Delta \mathrm{T}-1$ anomaly strength is about $433 \mathrm{nT}, \Delta \mathrm{T}-2$ anomaly strength is about $584 \mathrm{nT}$. The reduced to the pole data After upward continuation $500 \mathrm{~m}$, two anomalies disappeared, indicating that the two anomalies are shallow anomaly. According to the district physical properties and geological data, inferred that the cause of the two secondary magnetic anomalies source are shallow depth, so it should be caused by magnetic body in Tianshengba group.

The result of the AMT 2-D inversion display that sectional layer is better, the whole section is syncline structure characteristics, which coincides with the previous thought of Hongnipo syncline structure in the area. Simultaneously, AMT data inversion result reflected that the basic characteristics in this area is mainly low - high - low - high, which coincides with the basic physical characteristics of stratum in the survey area. We inferred a faults $\mathrm{F} 1$ at section $2250 \mathrm{~m}$ to the deep, the fault divided M163-1 into two abnormal; The resistivity is lower from shallow to deep in the section $4800 \mathrm{~m} \sim 6800 \mathrm{~m}$, which is about $1 \sim 15 \Omega \cdot \mathrm{m}$, inferred that it is low resistance fracture zone and water filling. According to Fig. 2, below two magnetic anomalies has two relatively high resistivity anomalies correspondingly, resistivity is about 50 to $160 \Omega \cdot \mathrm{m}$, showing in the Res-1 with Res2 of Fig. 2. According to the district physical properties and geological data, Tianshengba group is one of the iron and copper mine stratum and it's resistivity is relatively high; Xinqiao group does not contain mine and it's resistivity is relatively high. So inferred that the two relatively high resistivity anomaly is ore containing anomaly. 


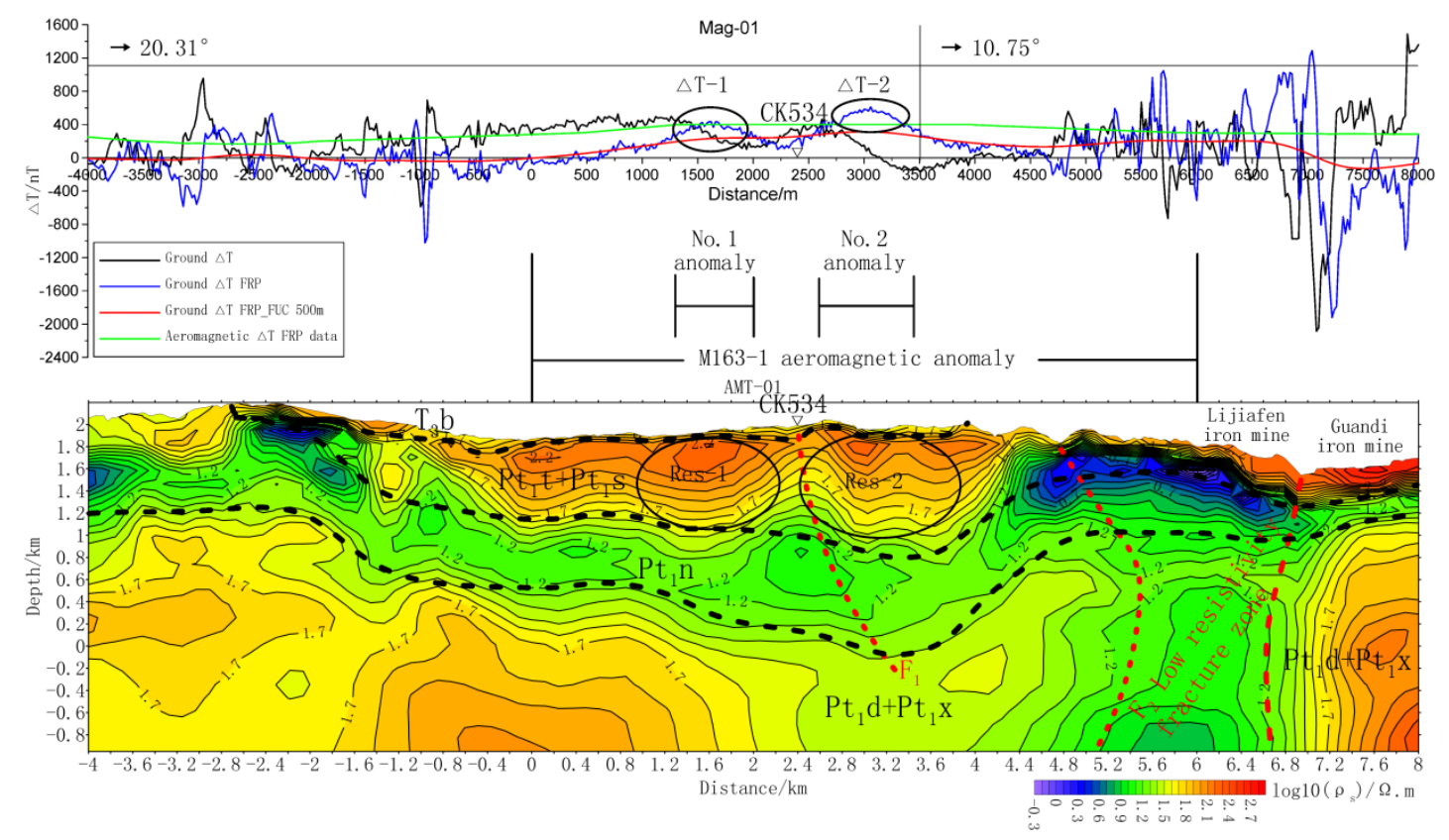

Fig. 2. magnetic and AMT profile comprehensive anomaly interpretation

Notably, the Previous person arranged a $604.14 \mathrm{~m}$ deep drilling (CK534) in the center of the aeromagnetic anomaly position, to verify M163-1 aeromagnetic anomalies,it found mineral,such as magneitet, pyrite, chalcopyrite, local grade is up to $10 \%$, but the overall grade is limited, so the result shows that the magnetic anomaly reflects the concealed and magnetite content alteration gabbro, drilling position is shown in Fig. 1 and figure Fig. 2. By comprehensively analyzing the ground high-precision magnetic survey and AMT measurement results and geological data, it can be seen that the drilling is located in the fault zone, and is not really in the center of magnetic anomaly, but in the middle of the two secondary magnetic anomaly, the place that is the most weak magnetic, and the ground magnetic survey anomaly intensity is about $100 \sim 200 \mathrm{nT}$. So, inferred that the drilling should be arranged unreasonable, which leads to the failure of finding ore of industrial grade. Magnetic anomaly on either side of the drilling is $200 \sim 500 \mathrm{nT}$ higher than magnetic anomaly in the drilling,therefore, it can be inferred that magnetic anomaly on either side of the drilling possible is the magnetic anomaly containing ore .

\section{IV . CONCLUSION}

The ground high-precision magnetic survey results show that M163-1 aeromagnetic anomaly is resulted by the combinenation of regional anomaly with two secondary anomaly.

AMT results shows that there exists a relatively wide and flat syncline structure in the region; Basic electrical structure characteristics mainly is low - high - low - high.
AMT and ground high-precision magnetic method provide new favorable evidence for ore-prospecting of old M163-1 aeromagnetic anomaly.

The ore-bearing potential is larger in the two anomalies areas, which are the favorable Prospecting prospect areas.

\section{REFERENCE}

[1] Chen G W, Xia B. Study on the Genesis of Lala Copper Deposit, Sichuan Province $[\mathrm{J}]$. Bulletin of Mineralogy Petrology \& Geochemistry, 2001, 20(01): 42-44.

[2] Feng-Chun Li, Sun Y. Characteristics and Significance of The La-la Copper Deposit in Sichuan Province[J]. Sichuan Nonferrous Metals, 2009(2):18-23.

[3] Xiao Y F, Yan S, Zhao Z Q, et al. Modes of occurrence of useful associated components in the Lala copper deposit,Sichuan Provice[J]. Geology in China, 2010, 37(2):463-469.

[4] [Qin S Q, Yu-Ping H U, Zhao X P. Application of Audio Magnetotelluric(AMT) for Metal Mine exploration[J].Journal of Yangtze University, 2011.

[5] Tang J, Fan J F. Application of integrated geophysical methods in the Lipuwu aeromagnetic anomaly inspection[J].Mineral Exploration, 2011.

[6] Wang Wenwen. The geophysical application research on deep concealed ore deposit prediction in LALA coppermines and it's peripheral area[D]. Chengdu:Chengdu university of technology, 2011:8-14.

[7] Zhou J, Wang X B, Yang D B, et al. The application of integrated geophysical methods to aeromagnetic anomaly inspection of Huidong area[J]. Geology in China, 2013, 40(4):1290-1297.

[8] Luo Yuquan,Deng Wenxun. The result report of Lala copper model geophysical and geochemical method application,prospecting model and prediction research in Huili,Sichuan province[R]. Chengdu:Comprehensive research team,Geophysical Exploring Team of Sichuan bureau of geology and mineral, 1990.4-1993.8. 